Pacific

Journal of

Mathematics

HARNACK INEQUALITY FOR NONDIVERGENT ELLIPTIC OPERATORS ON RIEMANNIAN MANIFOLDS

SEICK KIM

Volume 213 No. 2

February 2004 


\title{
HARNACK INEQUALITY FOR NONDIVERGENT ELLIPTIC OPERATORS ON RIEMANNIAN MANIFOLDS
}

\author{
SEICK KIM
}

\begin{abstract}
We consider second-order linear elliptic operators of nondivergence type which are intrinsically defined on Riemannian manifolds. Cabré proved a global Krylov-Safonov Harnack inequality under the assumption that the sectional curvature is nonnegative. We improve Cabré's result and, as a consequence, we give another proof to the Harnack inequality of Yau for positive harmonic functions on Riemannian manifolds with nonnegative Ricci curvature using the nondivergence structure of the Laplace operator.
\end{abstract}

\section{Introduction and main results.}

In this article we study Harnack inequalities for solutions of second-order elliptic equations on Riemannian manifolds. The Harnack inequality is well understood for divergence form operators on manifolds satisfying certain properties, namely the volume doubling property and the weak Poincaré inequality; see $[\mathbf{6}, \mathbf{1 0}]$ and $[\mathbf{1 1}]$. For example, the above two conditions hold for manifolds with nonnegative Ricci curvature. In fact, Grigor'yan [6] and Saloff-Coste $[\mathbf{1 1}]$ proved that the volume doubling property and the weak Poincaré inequality imply the Harnack inequality for solutions to divergence type parabolic equations. Furthermore, Saloff-Coste [11] showed that the above two conditions are equivalent to the Harnack inequality for the heat operator.

Recently, Cabré [1] considered nondivergence type elliptic operators and proved the Harnack inequality on manifolds with nonnegative sectional curvature; see below for the definition of nondivergent elliptic operators. His result is an extension of the Euclidean Krylov-Safonov Harnack inequality ([7] and [9]). Compared to the Harnack inequality for divergent operators, the hypothesis in the Harnack inequality of Cabré is not completely optimal and we improve it here. For example, the Laplace operator has both divergent and nondivergent structure and it is well-known that positive harmonic functions on Riemannian manifolds with nonnegative Ricci curvature satisfy the Harnack inequality, which was earlier proved by Yau [15]. Since the assumption in the Harnack inequality of Cabré requires the underlying 
manifold to have nonnegative sectional curvature, it does not directly imply the Harnack inequality of Yau.

The motivation for our work was to give another proof of Yau's Harnack inequality using the nondivergent structure of the Laplace operator; and by doing so, we expected to generalize Cabré's result. We found a sufficient condition in terms of the distance function on the underlying Riemannian manifold, which implies a global Krylov-Safonov Harnack inequality for solutions of uniformly elliptic equations of nondivergence type. We show that our result implies the Harnack inequality of Yau, and we will provide examples of such Riemannian manifolds, using the curvature assumption. Our examples strictly include the manifolds with nonnegative sectional curvature, which means that our result generalizes that of Cabré $[\mathbf{1}]$.

Let us begin with the definition of nondivergent elliptic operators on Riemannian manifolds. Let $(M, g)$ be a smooth, complete, noncompact Riemannian manifold of dimension $n$. For any $x \in M$, we denote by $T_{x} M$ the tangent space of $M$ at $x$. For any $x \in M$, let $A_{x}$ be a positive definite symmetric endomorphism of $T_{x} M$. We assume that

$$
\lambda|X|^{2} \leq\left\langle A_{x} X, X\right\rangle \leq \Lambda|X|^{2} \quad \forall x \in M, \quad \forall X \in T_{x} M,
$$

for some positive constants $\lambda$ and $\Lambda$. Here $\langle X, Y\rangle=g(X, Y)$ and $|X|^{2}=$ $\langle X, X\rangle$. We consider a second-order, linear, uniformly elliptic operator $L$ defined by

$$
L u=\operatorname{tr}\left(A_{x} \circ D^{2} u\right)=\operatorname{tr}\left\{X \mapsto A_{x} \nabla_{X} \nabla u\right\},
$$

where $\circ$ denotes composition of endomorphisms, tr is the trace, and $D^{2} u$ denote the Hessian of the function $u$. We recall that the Hessian of $u$ at $x \in M$ is the endomorphism of $T_{x} M$ defined by

$$
D^{2} u \cdot X=\nabla_{X} \nabla u
$$

where $\nabla u(x) \in T_{x} M$ is the gradient of $u$ at $x$. It may seem that our definition of nondivergent elliptic operator differs from that of Cabré [1], but since

$$
L u=\operatorname{tr}\left(A_{x} \circ D^{2} u\right)=\operatorname{tr}\left(D^{2} u \circ A_{x}\right)=\operatorname{tr}\left\{X \mapsto \nabla_{A_{x} X} \nabla u\right\},
$$

our definition of $L u$ coincides with the one that originally appeared in Cabré [1]. We point out that Stroock also used the convention (2) of nondivergent elliptic operators in his recent article [14].

In contrast, divergent operators in manifolds are of the form

$$
\mathcal{L} u=\operatorname{div}\left(A_{x} \nabla u\right),
$$

where div denotes the divergence. The Laplace operator has both divergent and nondivergent structure in the sense that $\Delta u$ can be viewed as either $\Delta u=\operatorname{div}(\nabla u)$ or $\Delta u=\operatorname{tr}\left(D^{2} u\right)$.

Let $p$ be a fixed point in $M$ and let $d_{p}(x)$ be the distance function defined by $d_{p}(x)=d(p, x)$, where $d(p, x)$ is the geodesic distance between $p$ and $x$. 
Recall that $d_{p}$ is smooth on $M \backslash(\operatorname{Cut}(p) \cup\{p\})$, where $\operatorname{Cut}(p)$ is the cut locus of $p$; see e.g., [12] and [13]. We assume that $M$ satisfies the following conditions:

$$
\begin{aligned}
& \Delta d_{p}(x) \leq \frac{n-1}{d_{p}(x)} \quad \text { for } \quad x \notin \operatorname{Cut}(p) \cup\{p\} \quad \forall p \in M \quad \text { and } \\
& L d_{p}(x) \leq \frac{a_{L}}{d_{p}(x)} \quad \text { for } \quad x \notin \operatorname{Cut}(p) \cup\{p\} \quad \forall p \in M,
\end{aligned}
$$

where $a_{L}$ is a constant which may depend on the given operator $L$.

By the Laplace comparison theorem (see e.g., [12] and [13]), any manifold with nonnegative Ricci curvature satisfies Condition (3). We shall give examples of manifolds satisfying Condition (4) shortly.

Let us state our main theorem. We would like to mention that the constant $C$ which appears in the following main result does not depend on the smoothness of the coefficients $A_{x}$. Indeed, it is one of the most important features in Moser and Krylov-Safonov type Harnack inequalities, including that of Cabré [1], which remain valid for operators with bounded measurable coefficients.

Theorem 1.1. Assume that $M$ satisfies Conditions (3) and (4). Let $u$ be a smooth function in a ball $B_{2 R}$ satisfying $u \geq 0$ in $B_{2 R}$. Then

$$
\sup _{B_{R}} u \leq C\left\{\inf _{B_{R}} u+\frac{R^{2}}{\left|B_{2 R}\right|^{1 / n}}\|L u\|_{L^{n}\left(B_{2 R}\right)}\right\},
$$

where $C$ is a constant depending only on $\lambda, \Lambda, n$ and $a_{L}$.

Here, $B_{2 R}$ is any geodesic ball of radius $2 R, B_{R}$ is the geodesic ball of radius $R$ concentric with $B_{2 R}$, and $\left|B_{2 R}\right|$ is the volume of $B_{2 R}$. As an immediate consequence, we obtain the following Liouville property for solutions of $L u=0$ in $M$ :

Corollary 1.2. Let $u$ be a smooth solution of $L u=0$ in $M$ that is bounded from below. Then $u$ is constant.

We now give examples of manifolds satisfying (3) and (4). This can be done in a most transparent way by stating it in terms of Pucci's extremal operator. For a symmetric endomorphism $S$ on $T_{x} M$, we define

$$
\mathcal{M}^{-}[S, \lambda, \Lambda]=\mathcal{M}^{-}[S]=\lambda \sum_{\kappa_{i}>0} \kappa_{i}+\Lambda \sum_{\kappa_{i}<0} \kappa_{i},
$$

where $\kappa_{i}=\kappa_{i}(S)$ are the eigenvalues of $S$. Suppose $M$ satisfies the following curvature assumption: For any unit vector $e$ in $T_{x} M$ and for all $x \in M$,

$$
\mathcal{M}^{-}[R(e)] \geq 0,
$$

where $R(e)$ is the Ricci transformation on $T_{x} M$; see Sec. 2 for its definition. Note that the inequality $\mathcal{M}^{-}[R(e)] \leq \operatorname{tr}\left(A_{x} \circ R(e)\right)$ holds provided that 
$A_{x}$ satisfies the ellipticity condition (1). Thus, by taking $A_{x}=\lambda \mathrm{Id}$, we find that Condition (5) implies that $M$ has nonnegative Ricci curvature and hence, by the Laplace comparison theorem (see [12] and [13]), it implies (3). We shall see in the next section that (5) also implies Condition (4) with $a_{L}=(n-1) \Lambda$; see Lemma 2.1 below. Then, the next corollary will follow immediately.

Corollary 1.3. Assume that $M$ satisfies the curvature condition (5). Let $u$ be a smooth function in a ball $B_{2 R}$ satisfying $u \geq 0$ in $B_{2 R}$. Then

$$
\sup _{B_{R}} u \leq C\left\{\inf _{B_{R}} u+\frac{R^{2}}{\left|B_{2 R}\right|^{1 / n}}\|L u\|_{L^{n}\left(B_{2 R}\right)}\right\},
$$

where $C$ is a universal constant; i.e., a constant depending on the ellipticity constants $\lambda, \Lambda$ and the dimension $n$ only.

Note that in the case when $L=\Delta$, Condition (5) is just to say that the Ricci curvature is nonnegative. Hence, Theorem 1.1 and Corollary 1.2, respectively, imply the Harnack inequality and the Liouville property by Yau [15]. Moreover, since Condition (5) holds trivially for manifolds with nonnegative sectional curvature, our result also generalizes the Harnack inequality of Cabré [1].

\section{Preliminaries.}

Recall, for $x \in M$, the exponential map $\exp _{x}: T_{x} M \rightarrow M$. For $X \in T_{x} M$ with $|X|=1, \gamma(t)=\exp _{x}(t X)$ is the unique unit speed geodesic that starts from $x$ and goes in the direction of $X$. Let

$$
t_{0}=\sup \{t>0: \gamma \text { is the unique minimal geodesic joining } x \text { and } \gamma(t)\} .
$$

If $t_{0}<\infty$, then $\gamma\left(t_{0}\right)$ is called a cut point of $x$. We denote

$$
\operatorname{Cut}(x)=\text { set of all cut points of } x \text {. }
$$

If we denote $S_{x}=\left\{X \in T_{x} M:|X|=1\right\}$, it is clear that for any $X \in S_{x}$, there can be at most one cut point on the geodesic $\exp _{x}(t X), t>0$. If $\exp _{x}\left(t_{0} X\right)=y$ is a cut point of $x$ then we set $\mu(X)=d(x, y)$, the geodesic distance between $x$ and $y$. If there is no cut point, we set $\mu(X)=\infty$. Define

$$
E_{x}:=\left\{t X: 0 \leq t<\mu(X), X \in S_{x}\right\} .
$$

Then, it can be shown that $\exp _{x}: E_{x} \rightarrow \exp _{x}\left(E_{x}\right)$ is a diffeomorphism and, also that $\operatorname{Cut}(x)$ has $n$-dimensional measure zero; see [12] and [13].

Let the Riemannian curvature tensor be defined by

$$
R(X, Y) Z=\nabla_{X} \nabla_{Y} Z-\nabla_{Y} \nabla_{X} Z-\nabla_{[X, Y]} Z,
$$

where $\nabla$ is the Levi-Civita connection. For a unit vector $e \in T_{x} M, R(e)$ will denote the Ricci transformation of $T_{x} M$ into itself given by $R(e) X:=$ 
$R(X, e) e$. The Morse index form $I(\cdot, \cdot)$ is defined by

$$
I(X, Y)=I_{0}^{\ell}(X, Y)=\int_{0}^{\ell}\left\{\left\langle\nabla_{\gamma^{\prime}} X, \nabla_{\gamma^{\prime}} Y\right\rangle-\left\langle R\left(\gamma^{\prime}, X\right) Y, \gamma^{\prime}\right\rangle\right\} d t,
$$

where $\gamma:[0, \ell] \rightarrow M$ is a geodesic parametrized by arc length and $X, Y$ are piecewise smooth vector fields along $\gamma$.

Now, we show that Condition (5) implies (4) with $a_{L}=(n-1) \Lambda$.

Lemma 2.1. Let $M$ be an n-dimensional complete Riemannian manifold satisfying the curvature condition (5). Let $d_{p}$ be the distance function from a fixed point $p \in M$. If $x \notin \operatorname{Cut}(p) \cup\{p\}$, then

$$
L d_{p}(x) \leq \frac{a_{L}}{d_{p}(x)}, \quad \text { where } a_{L}=(n-1) \Lambda .
$$

Proof. Let $\gamma:[0, \rho] \rightarrow M$ be the minimal geodesic parametrized by arc length joining $\gamma(0)=p$ and $\gamma(\rho)=x$. Choose an orthonormal basis $\left\{e_{i}\right\}_{i=1}^{n}$ on $T_{x} M$ such that $e_{1}=\gamma^{\prime}(\rho)$ and $\left\{e_{i}\right\}_{i=1}^{n}$ are eigenvectors of $D^{2} d_{p}$ on $T_{x} M$. We extend $\left\{e_{i}\right\}_{i=1}^{n}$ to $\left\{e_{i}(t)\right\}_{i=1}^{n}$ on $t \in[0, \rho]$ by parallel translation. Let $X_{i}$, $i=2, \ldots, n$, be the Jacobi fields along $\gamma$ such that $X_{i}(0)=0, X_{i}(\rho)=e_{i}$ and $\left[X_{i}, \gamma^{\prime}\right]=0$. Then $\left\langle D^{2} d_{p}\left(e_{i}\right), e_{i}\right\rangle=\left\langle\nabla_{\gamma^{\prime}} X_{i}, X_{i}\right\rangle(\rho)=I\left(X_{i}, X_{i}\right)$. Let $Y_{i}=f(t) e_{i}(t)$, where $f(t)=\frac{t}{\rho}$.

Since a Jacobi field minimizes the index form among all vector fields along the same geodesic with the same boundary data, we have $I\left(X_{i}, X_{i}\right) \leq$ $I\left(Y_{i}, Y_{i}\right)$. Denote $a_{i j}:=\left\langle A_{x} e_{i}, e_{j}\right\rangle$. Then

$$
L d_{p}(x)=\sum_{i=2}^{n} a_{i i}\left\langle D^{2} d_{p}\left(e_{i}\right), e_{i}\right\rangle=\sum_{i=2}^{n} a_{i i} I\left(X_{i}, X_{i}\right) \leq \sum_{i=2}^{n} a_{i i} I\left(Y_{i}, Y_{i}\right) .
$$

On the other hand,

$$
\begin{aligned}
\sum_{i=2}^{n} a_{i i} I\left(Y_{i}, Y_{i}\right) & =\sum_{i=2}^{n} a_{i i} \int_{0}^{\rho}\left|f^{\prime}\right|^{2}-\int_{0}^{\rho} f^{2} \sum_{i=2}^{n} a_{i i}\left\langle R\left(\gamma^{\prime}, e_{i}\right) e_{i}, \gamma^{\prime}\right\rangle \\
& \leq \sum_{i=2}^{n} a_{i i} \int_{0}^{\rho}\left|f^{\prime}\right|^{2}-\int_{0}^{\rho} f^{2} \mathcal{M}^{-}\left[R\left(\gamma^{\prime}\right)\right] \\
& \leq \frac{(n-1) \Lambda}{\rho} .
\end{aligned}
$$

This completes the proof.

Observe that in the case when $\lambda=\Lambda=1$ (i.e., $L=\Delta$ ), Condition (5) reduces to the nonnegative Ricci curvature assumption on $M$. Also, note that if the sectional curvature of $M$ is nonnegative, then (5) holds trivially.

Let us recall that, if $\phi$ is a smooth map from $M$ to another Riemannian manifold $N$, the Jacobian of $\phi$ is the absolute value of the determinant of the differential of $\phi$, that is, $\operatorname{Jac} \phi(x):=|\operatorname{det} d \phi(x)|$. This determinant is 
computed when expressing $d \phi(x)$ in an orthonormal basis of $T_{x} M$ and an orthonormal basis of $T_{\phi(x)} N$, and hence it is defined up to a sign. Its absolute value, Jac $\phi(x)$, is therefore well-defined. The following is the area formula on $M$, which follows easily from the area formula in Euclidean space using a partition of unity; see [1]: For any smooth map $\phi$ from $M$ to $M$ and any measurable subset $E$ of $M$, we have

$$
\int_{E} \operatorname{Jac} \phi(x) d V(x)=\int_{M} \mathcal{H}^{0}\left[E \cap \phi^{-1}(y)\right] d V(y),
$$

where $\mathcal{H}^{0}$ is the counting measure.

The following lemma is quoted from [1, Lemma 3.2].

Lemma 2.2 (Cabré). Let $v$ be a smooth function in an open set $\Omega$ of $M$. Consider the map $\phi$ from $\Omega$ to $M$ defined by

$$
\phi(p)=\exp _{p} \nabla v(p) .
$$

Let $x \in \Omega$ and suppose that $\nabla v(x) \in E_{x}$. Set $y=\phi(x)$. Then

$$
\operatorname{Jac} \phi(x)=\operatorname{Jac}_{\exp }(\nabla v(x)) \cdot\left|\operatorname{det} D^{2}\left(v+d_{y}^{2} / 2\right)(x)\right|,
$$

where $\operatorname{Jac} \exp _{x}(\nabla v(x))$ denotes the Jacobian of $\exp _{x}$, a map from $T_{x} M$ to $M$ at the point $\nabla v(x) \in T_{x} M$.

In the normal polar coordinates $(r, \theta)$, the area element $J(r, \theta) d \theta$ of the geodesic sphere $\partial B_{r}(x)$ of radius $r$ centered at $x$ is given by $r^{n-1} A(r, \theta) d \theta$, where $A(r, \theta)$ is the Jacobian of the map $\exp _{x}$ at $r \theta \in T_{x} M$. Assume that $M$ satisfies Condition (3). For $y=(r, \theta)$ not in the cut locus of $x$, we have the inequality $\frac{\partial}{\partial r} \ln J(r, \theta)=\Delta r \leq \frac{n-1}{r}$; see [8]. By integrating this, we find $A(r, \theta)=r^{1-n} J(r, \theta)$ is a nonincreasing function of $r$ and, in particular, $A(r, \theta) \leq 1$ since $\lim _{r \rightarrow 0} A(r, \theta)=1$. Hence, Lemma 2.2 implies

$$
\operatorname{Jac} \phi(x) \leq\left|\operatorname{det} D^{2}\left(v+d_{y}^{2} / 2\right)(x)\right| .
$$

Also, from the above observation, it follows that $r^{-n}\left|B_{r}\right|$ is nonincreasing in $r$; see e.g., [8] and [13]. This is generally known as Bishop's volume comparison theorem. We state it as a lemma.

Lemma 2.3 (Bishop). Let $M$ be an $n$-dimensional Riemannian manifold satisfying (3). For any $x \in M,\left|B_{R}(x)\right| / R^{n}$ is nonincreasing with respect to $R$. In particular,

$$
\left|B_{R}(x)\right| /\left|B_{r}(x)\right| \leq R^{n} / r^{n} \quad \text { if } \quad 0<r<R,
$$

and $M$ satisfies the volume doubling property; i.e., $\left|B_{2 R}(x)\right| \leq 2^{n}\left|B_{R}(x)\right|$. 


\section{Proof of Harnack inequality.}

Throughout the entire section, we shall assume that $M$ satisfies (3) and (4). We will closely follow the outline of Cabré's proof of the Harnack inequality in [1], which in turn carried over the basic scheme in the book by Caffarelli and Cabré [3]; see also [2]. Our goal is to establish Lemma 3.1 below. It corresponds to [1, Lemma 5.1], in the proof of which Cabré used the assumption that the underlying manifold $M$ has nonnegative sectional curvature. If one investigates his arguments carefully, it will turn out that, besides [1, Lemma 5.1], the only geometrical property of $M$ he used in proving the subsequent lemmas in Sec. 6 and Sec. 7 of [1] is the volume growth condition (8). Therefore, once we prove Lemma 3.1, the proof of Theorem 1.1 follows from the one in [1] verbatim since, by Lemma 2.3 of the previous section, Property (8) holds for any manifold satisfying Condition (3).

Lemma 3.1. Let $u$ be a smooth function in a ball $B_{7 R}$ satisfying

$$
L u \leq f \text { in } B_{7 R}, \quad u \geq 0 \text { in } B_{7 R}, \quad \inf _{B_{2 R}} u \leq 1,
$$

and

$$
\frac{R^{2}}{\left|B_{7 R}\right|^{1 / n}}\|f\|_{L^{n}\left(B_{7 R}\right)} \leq \varepsilon_{\delta} .
$$

Then, for any $0<\delta<1$,

$$
\frac{\left|\left\{u \leq M_{\delta}\right\} \cap B_{\delta R}\right|}{\left|B_{7 R}\right|} \geq \mu_{\delta},
$$

where $\varepsilon_{\delta}, 0<\mu_{\delta}<1$ and $M_{\delta}>1$ are positive constants depending on $\delta$.

We need a series of lemmas to prove Lemma 3.1 above. The next lemma is a modification of [1, Lemma 4.1], where Cabré assumed the nonnegativity of the sectional curvature to get the same conclusion. We point out that the Krylov-Safonov theory in Euclidean space is based on the AlexandrovBakelman-Pucci (ABP) estimate, which in turn is based on the existence of linear functions with constant gradient on Euclidean space. Such functions do not exist in most Riemannian manifolds and were replaced in [1] by paraboloids.

Lemma 3.2. Let $u$ be a smooth function in a ball $B_{7 R}:=B_{7 R}(p)$ satisfying $u \geq 0$ in $B_{7 R} \backslash B_{5 R}$ and $\inf _{B_{2 R}} u \leq 1$. Then

$$
\left|B_{R}\right| \leq \frac{1}{(n \lambda)^{n}} \int_{\{u \leq 6\} \cap B_{5 R}}\left\{\left(R^{2} L u+a_{L}+\Lambda\right)^{+}\right\}^{n} d V .
$$

Here, we denote by $f^{+}$the positive part of a function, i.e., $f^{+}=\max (f, 0)$. 
Proof. For any $y \in B_{R}$ we consider the continuous function

$$
w_{y}:=R^{2} u+\frac{1}{2} d_{y}^{2} .
$$

We have that $\inf _{B_{2 R}} w_{y} \leq R^{2}+(3 R)^{2} / 2=11 R^{2} / 2$. In $B_{7 R} \backslash B_{5 R}$ we have, since $u \geq 0$ here, $w_{y} \geq(4 R)^{2} / 2>11 R^{2} / 2$. We conclude that the minimum of $w_{y}$ in $\overline{B_{5 R}}$ is achieved at some point of $B_{5 R}$, which is also a minimum of $w_{y}$ in $B_{7 R}$. That is,

$$
\inf _{B_{7 R}} w_{y}=\inf _{B_{5 R}} w_{y}=w_{y}(x),
$$

for some $x \in B_{5 R}$. It is not hard to see that $y=\exp _{x} \nabla\left(R^{2} u\right)(x)$; see [1, pp. 637-638]. We are therefore led to consider the smooth map from $B_{7 R}$ to $M$

$$
\phi(z)=\exp _{z} \nabla\left(R^{2} u\right)(z)
$$

and the measurable set

$$
E:=\left\{x \in B_{5 R}: \exists y \in B_{R} \text { such that } w_{y}(x)=\inf _{B_{7 R}} w_{y}\right\} .
$$

We have proved that for any $y \in B_{R}$ there is at least one $x \in E$ such that $\phi(x)=y$. The area formula (6) gives

$$
\left|B_{R}\right| \leq \int_{M} \mathcal{H}^{0}\left[E \cap \phi^{-1}(y)\right] d V(y)=\int_{E}(\operatorname{Jac} \phi) d V .
$$

We claim that $\operatorname{Jac} \phi(x) \leq(n \lambda)^{-n}\left\{R^{2} L u(x)+a_{L}+\Lambda\right\}^{n}$ for any $x \in E$.

Let $x \in E$ and take $y \in B_{R}$ such that $w_{y}(x)=\inf _{B_{7 R}} w_{y}$. If $x$ is not a cut point of $y$, then by (7) we find

$$
\operatorname{Jac} \phi(x) \leq\left|\operatorname{det} D^{2}\left(R^{2} u+d_{y}^{2} / 2\right)(x)\right|=\left|\operatorname{det} D^{2} w_{y}(x)\right| .
$$

Since $w_{y}$ achieves its minimum at $x, D^{2} w_{y}(x) \geq 0$. Here we used $\leq$ to denote the usual order between symmetric endomorphisms. Therefore, by using the well-known inequality

$$
\operatorname{det} A \cdot \operatorname{det} B \leq\left\{n^{-1} \operatorname{tr}(A \circ B)\right\}^{n}, \quad A, B \text { symmetric } \geq 0,
$$
we conclude

$$
\begin{aligned}
\operatorname{Jac} \phi(x) & \leq \operatorname{det} D^{2} w_{y}(x) \leq \frac{1}{\lambda^{n}} \operatorname{det} A_{x} \cdot \operatorname{det} D^{2} w_{y}(x) \\
& \leq \frac{1}{(n \lambda)^{n}}\left\{\operatorname{tr}\left(A_{x} \circ D^{2} w_{y}(x)\right)\right\}^{n} \\
& =\frac{1}{(n \lambda)^{n}}\left\{L w_{y}(x)\right\}^{n} \\
& =\frac{1}{(n \lambda)^{n}}\left\{R^{2} L u(x)+L\left(d_{y}^{2} / 2\right)(x)\right\}^{n} \\
& \leq \frac{1}{(n \lambda)^{n}}\left\{R^{2} L u(x)+a_{L}+\Lambda\right\}^{n}
\end{aligned}
$$


where we used

$$
L\left(d_{y}^{2} / 2\right)=d_{y} L d_{y}+\left\langle A \nabla d_{y}, \nabla d_{y}\right\rangle \leq a_{L}+\Lambda\left|\nabla d_{y}\right|^{2}
$$

in the last step to get $L\left(d_{y}^{2} / 2\right) \leq a_{L}+\Lambda$.

We also have to consider the other case, namely the case when $x$ is a cut point of $y$. In general, this kind of situation is easily overcome. Indeed, we will make use of upper barrier technique due to Calabi [4]; see also [5]. Since $y=\exp _{x} \nabla\left(R^{2} u\right)(x), x$ is not a cut point of $y_{s}=\phi_{s}(x):=\exp _{x} \nabla\left(s R^{2} u\right)(x)$, for all $0 \leq s<1$. By continuity, $\operatorname{Jac} \phi(x)=\lim _{s \rightarrow 1} \operatorname{Jac} \phi_{s}(x)$. As before,

$$
\operatorname{Jac} \phi_{s}(x) \leq\left|\operatorname{det} D^{2}\left(s R^{2} u+d_{y_{s}}^{2} / 2\right)(x)\right| \text {. }
$$

Also,

$$
\begin{aligned}
\varliminf_{s \rightarrow 1}\left|\operatorname{det} D^{2}\left(s R^{2} u+d_{y_{s}}^{2} / 2\right)(x)\right| & ={\underline{\varliminf_{s \rightarrow 1}}}_{s \rightarrow 1}\left|\operatorname{det} D^{2}\left(R^{2} u+d_{y_{s}}^{2} / 2\right)(x)\right| \\
& =\underline{\lim }_{s \rightarrow 1}\left|\operatorname{det} D^{2} w_{y_{s}}(x)\right| .
\end{aligned}
$$

By the triangle inequality, we have

$$
R^{2} u+\frac{1}{2}\left[d_{y_{s}}+d\left(y_{s}, y\right)\right]^{2} \geq w_{y} .
$$

Note that the equality holds at $x$. Since the function

$$
R^{2} u+\frac{1}{2}\left[d_{y_{s}}+d\left(y_{s}, y\right)\right]^{2}=w_{y_{s}}+d\left(y_{s}, y\right) d_{y_{s}}+\frac{1}{2} d\left(y_{s}, y\right)^{2}
$$

is smooth near $x$ and has a local minimum at $x$ (recall that $w_{y}$ has a minimum at $x)$, its Hessian at $x$ is nonnegative definite. Let $-k^{2}(k>0)$ be a lower bound of sectional curvature along the minimal geodesic joining $x$ and $y$. By the Hessian comparison theorem (see, e.g., [12] and [13]),

$$
D^{2} d_{y_{s}}(x) \leq k \operatorname{coth}\left(k d\left(x, y_{s}\right)\right) \mathrm{Id} \leq N \mathrm{Id},
$$

uniformly in $s \in\left[\frac{1}{2}, 1\right)$ for some number $N$. Therefore, for all $s \in\left[\frac{1}{2}, 1\right)$,

$$
0 \leq D^{2} w_{y_{s}}(x)+d\left(y_{s}, y\right) D^{2} d_{y_{s}}(x) \leq D^{2} w_{y_{s}}(x)+N d\left(y_{s}, y\right) \operatorname{Id} .
$$

In particular, $D^{2} w_{y_{s}}(x)+N d\left(y_{s}, y\right)$ Id is nonnegative definite. As before, using inequality (12), we obtain $\forall s \in\left[\frac{1}{2}, 1\right)$

$$
\begin{aligned}
& \operatorname{det}\left(D^{2} w_{y_{s}}(x)+N d\left(y_{s}, y\right) \mathrm{Id}\right) \\
& \leq \frac{1}{\lambda^{n}}\left\{n^{-1} L w_{y_{s}}(x)+N d\left(y_{s}, y\right) \Lambda\right\}^{n} \\
& \leq \frac{1}{\lambda^{n}}\left\{n^{-1}\left(R^{2} L u(x)+a_{L}+\Lambda\right)+N d\left(y_{s}, y\right) \Lambda\right\}^{n} .
\end{aligned}
$$


Since $d\left(y_{s}, y\right) \rightarrow 0$ as $s \rightarrow 1$, we observe

$$
\begin{aligned}
0 & \leq \underline{\lim }_{s \rightarrow 1}\left|\operatorname{det} D^{2} w_{y_{s}}(x)\right|=\underline{\lim }_{s \rightarrow 1} \operatorname{det}\left(D^{2} w_{y_{s}}(x)+N d\left(y_{s}, y\right) \operatorname{Id}\right) \\
& \leq \frac{1}{\lambda^{n}} \underline{\lim }_{s \rightarrow 1}\left\{n^{-1}\left(R^{2} L u(x)+a_{L}+\Lambda\right)+N d\left(y_{s}, y\right) \Lambda\right\}^{n} \\
& =\frac{1}{(n \lambda)^{n}}\left\{R^{2} L u(x)+a_{L}+\Lambda\right\}^{n} .
\end{aligned}
$$

Putting these steps together, we finally obtain

$$
\operatorname{Jac} \phi(x) \leq \frac{1}{(n \lambda)^{n}}\left\{R^{2} L u(x)+a_{L}+\Lambda\right\}^{n} \quad \forall x \in E,
$$

which proves the claim. Obviously,

$$
\int_{E}(\operatorname{Jac} \phi) d V \leq \frac{1}{(n \lambda)^{n}} \int_{E}\left\{\left(R^{2} L u(x)+a_{L}+\Lambda\right)^{+}\right\}^{n} d V .
$$

At the beginning of the proof we have shown

$$
E \subset\{u \leq 11 / 2\} \cap B_{5 R} \subset\{u \leq 6\} \cap B_{5 R} .
$$

Now, the lemma follows from (11) and (13).

The following technical lemma, which consists of the construction of a barrier, will be used in the proof of Lemma 3.1. It corresponds to [1, Lemma 5.5] and [3, Lemma 4.1].

Lemma 3.3 (A barrier function). Let $p \in M, R>0$, and $0<\delta<1$. There exists a continuous function $v_{\delta}$ in $B_{7 R}=B_{7 R}(p)$, smooth in $M \backslash$ $\operatorname{Cut}(p)$ and such that:

(a) $v_{\delta} \geq 0$ in $B_{7 R} \backslash B_{5 R}$,

(b) $v_{\delta} \leq 0$ in $B_{2 R}$,

(c) $R^{2} L v_{\delta}+a_{L}+\Lambda \leq 0$ a.e. in $\left(B_{5 R} \backslash B_{\delta R}\right)$,

(d) $R^{2} L v_{\delta} \leq C_{\delta}$ a.e. in $B_{5 R}$ and

(e) $v_{\delta} \geq-C_{\delta}$ in $B_{7 R}$,

for some positive constant $C_{\delta}$ depending on $\delta$.

Proof. We take $v_{\delta}=\psi_{\delta}\left(d_{p} / R\right)$, where $\psi_{\delta}$ is a smooth increasing function on $\mathbb{R}^{+}$such that $\psi_{\delta}^{\prime}(0)=0$ and $\psi_{\delta}(t)=\left(\frac{3}{5}\right)^{-\alpha}-\left(\frac{t}{5}\right)^{-\alpha}$ if $t \geq \delta$. The number $\alpha>1$ will be chosen later. Clearly, $v_{\delta}$ is continuous in $M$. Also, since $d_{p}$ is smooth outside $\operatorname{Cut}(p) \cup\{p\}$ and $\psi_{\delta}^{\prime}(0)=0$, $v_{\delta}$ is smooth in $M \backslash \operatorname{Cut}(p)$. Recall that $\operatorname{Cut}(p)$ is a closed set of measure zero.

Properties (a) and (b) are clear. For the rest of proof, we will denote $\rho:=d_{p} / R$. From the identity $L[\varphi(u)]=\varphi^{\prime}(u) L u+\varphi^{\prime \prime}(u)\langle A \nabla u, \nabla u\rangle$, we get

$$
\begin{aligned}
L v_{\delta} & =\frac{1}{R} \psi_{\delta}^{\prime}(\rho) L d_{p}+\frac{1}{R^{2}} \psi_{\delta}^{\prime \prime}(\rho)\left\langle A \nabla d_{p}, \nabla d_{p}\right\rangle \\
& =\frac{1}{R^{2}} \frac{\psi_{\delta}^{\prime}(\rho)}{\rho} d_{p} L d_{p}+\frac{1}{R^{2}} \psi_{\delta}^{\prime \prime}(\rho)\left\langle A \nabla d_{p}, \nabla d_{p}\right\rangle \quad \text { in } M \backslash \operatorname{Cut}(p) .
\end{aligned}
$$


For $\delta \leq \rho<5$, we have

$$
\psi_{\delta}^{\prime}(\rho)=\frac{\alpha}{5}\left(\frac{\rho}{5}\right)^{-\alpha-1} \quad \text { and } \quad \psi_{\delta}^{\prime \prime}(\rho)=-\frac{\alpha(\alpha+1)}{5^{2}}\left(\frac{\rho}{5}\right)^{-\alpha-2} .
$$

We recall that

$$
d_{p} L d_{p} \leq a_{L} \quad \text { and } \quad \lambda \leq\left\langle A \nabla d_{p}, \nabla d_{p}\right\rangle \leq \Lambda \quad \text { in } M \backslash \operatorname{Cut}(p) .
$$

Hence, in $\left(B_{5 R} \backslash B_{\delta R}\right) \backslash \operatorname{Cut}(p)$ we have

$$
R^{2} L v_{\delta} \leq \frac{\alpha}{25}\left(\frac{\rho}{5}\right)^{-\alpha-2}\left(a_{L}-(\alpha+1) \lambda\right)
$$

which can be made less than $-\left(a_{L}+\Lambda\right)$ if $\alpha$ is chosen large enough. This proves Property (c).

Also, (14), (15), and the assumptions on $\psi_{\delta}$ imply that in $B_{5 R} \backslash \operatorname{Cut}(p)$

$$
R^{2} L v_{\delta} \leq a_{L} \sup _{0<\rho<5} \frac{\psi_{\delta}^{\prime}(\rho)}{\rho}+\Lambda \sup _{0<\rho<5}\left|\psi_{\delta}^{\prime \prime}(\rho)\right|<+\infty,
$$

which proves Property (d). Property (e) is clear.

In the Proof of Lemma 3.1, we will apply Lemma 3.2 to $u+v_{\delta}$ with $v_{\delta}$ constructed in the proof of Lemma 3.3. Possible existence of the cut locus of $p$ may present some technical issue here; $u+v_{\delta}$ is not necessarily smooth in $B_{7 R}$. To overcome this difficulty, we will again stick to Cabré's approach in [1]. It is not hard to verify the following lemma, which asserts that one can approximate $v_{\delta}$ by a sequence of smooth functions; see [1, pp. 641-645].

Lemma 3.4 (Cabré). Let $p \in M, R>0$, and $\psi: \mathbb{R}^{+} \rightarrow \mathbb{R}$ be a smooth increasing function such that $\psi^{\prime}(0)=0$. Let $v=\psi \circ d_{p}$. Then there exist a smooth function $0 \leq \xi \leq 1$ in $M$ with $\xi \equiv 1$ in $B_{5 R}:=B_{5 R}(p)$ and $\operatorname{supp} \xi \subset B_{7 R}$ and a sequence $\left\{w_{k}\right\}_{k=1}^{\infty}$ of smooth functions in $M$ such that

$$
\begin{cases}w_{k} \rightarrow \xi v & \text { uniformly in } M, \\ L w_{k} \rightarrow L v & \text { a.e. in } B_{5 R}, \text { and } \\ D^{2} w_{k} \leq C \text { Id } & \text { in } M \text { for some constant } C \text { independent of } k .\end{cases}
$$

Now, we are ready to prove Lemma 3.1.

Proof of Lemma 3.1. Let $v_{\delta}$ be as in Lemma 3.3. Let $\left\{w_{k}\right\}$ be a sequence of smooth functions approximating $v_{\delta}$ in the way described in Lemma 3.4. Note that $u+v_{\delta} \geq 0$ in $B_{7 R} \backslash B_{5 R}$ and $\inf _{B_{2 R}}\left(u+v_{\delta}\right) \leq 1$. Replacing $u+w_{k}$ by $\left(u+w_{k}+\varepsilon_{k}\right) /\left(1+2 \varepsilon_{k}\right)$ for some sequence $0<\varepsilon_{k} \rightarrow 0$ as $k \rightarrow \infty$, we may assume that $u+w_{k}$ satisfies the hypothesis of Lemma 3.2, and hence (10) holds with $u$ replaced by $u+w_{k}$. Let $\varepsilon>0$ and note that $\left\{u+w_{k} \leq 6\right\} \cap B_{5 R} \subset\left\{u+v_{\delta} \leq 6+\varepsilon\right\} \cap B_{5 R}$ if $k$ is large enough, and that $\left\{\left(R^{2} L\left(u+w_{k}\right)+a_{L}+\Lambda\right)^{+}\right\}^{n}$ is uniformly bounded in $M$ independently of $k$ (since $D^{2} w_{k} \leq C \mathrm{Id}$ ). Letting $k$ tend to infinity and applying the dominated 
convergence theorem, we get that $u+v_{\delta}$ satisfies (10) with $\{u \leq 6\}$ replaced by $\left\{u+v_{\delta} \leq 6+\varepsilon\right\}$. Now letting $\varepsilon$ tend to zero, we obtain, since $L u \leq f$,

$$
\begin{aligned}
\left|B_{R}\right| & \leq \frac{1}{(n \lambda)^{n}} \int_{\left\{u+v_{\delta} \leq 6\right\} \cap B_{5 R}}\left\{\left(R^{2}\left(f+L v_{\delta}\right)+a_{L}+\Lambda\right)^{+}\right\}^{n} d V \\
& =\frac{1}{(n \lambda)^{n}}\left[\int_{E_{1}}+\int_{E_{2}}\left\{\left(R^{2}\left(f+L v_{\delta}\right)+a_{L}+\Lambda\right)^{+}\right\}^{n} d V\right],
\end{aligned}
$$

where $E_{1}=\left\{u+v_{\delta} \leq 6\right\} \cap\left(B_{5 R} \backslash B_{\delta R}\right)$ and $E_{2}=\left\{u+v_{\delta} \leq 6\right\} \cap B_{\delta R}$.

Using (c), (d) and (e) in Lemma 3.3 and (8), we get

$$
\left|B_{7 R}\right| \leq 7^{n}\left|B_{R}\right| \leq C_{\delta}^{\prime}\left\{R^{2 n}\|f\|_{L^{n}\left(B_{7 R}\right)}^{n}+\left|\left\{u \leq M_{\delta}\right\} \cap B_{\delta R}\right|\right\},
$$

for some constants $C_{\delta}^{\prime}$ and $M_{\delta}$ depending on $\delta$. We easily conclude that (9) follows.

Acknowledgment. The author wishes to express his thank to Professor Mikhail Safonov and Jiaping Wang for valuable discussions. The author would also like to thank the anonymous referee for helpful suggestions and comments.

\section{References}

[1] X. Cabré, Nondivergent elliptic equations on manifolds with nonnegative curvature, Comm. Pure Appl. Math., 50 (1997), 623-665, MR 98b:58157, Zbl 0878.58054.

[2] L.A. Caffarelli, Interior a priori estimates for solutions of fully nonlinear equations, Ann. of Math. (2), 130(1) (1989), 189-213, MR 90i:35046, Zbl 0692.35017.

[3] L.A. Caffarelli and X. Cabré, Fully Nonlinear Elliptic Equations, American Mathematical Society Colloquium Publications, 43, Amer. Math. Soc., Providence, RI, 1995, MR 96h:35046, Zbl 0834.35002.

[4] E. Calabi, An extension of E. Hopf's maximum principle with an application to Riemannian geometry, Duke Math. J., 25 (1957), 45-56, MR 19,1056e, Zbl 0079.11801.

[5] J. Cheeger, Critical points of distance functions and applications to geometry, Geometric Topology: Recent Developments, 1-38, Lecture Notes in Mathematics, 1504, Springer, Berlin, 1991, MR 94a:53075, Zbl 0771.53015.

[6] A.A. Grigor'yan, The heat equation on noncompact Riemannian manifolds (Russian), Mat. Sb., 182(1) (1991), 55-87; Math. USSR-Sb., 72(1) (1992), $47-77$ (English), MR 92h:58189, Zbl 0776.58035.

[7] N.V. Krylov and M.V. Safonov, A property of the solutions of parabolic equations with measurable coefficients (Russian), Izv. Akad. Nauk SSSR Ser. Mat., 44(1) (1980), 161-175; Math. USSR Izvestija, 16 (1981), 151-164 (English), MR 83c:35059, Zbl 0464.35035.

[8] P. Li, Lecture Notes on Geometric Analysis, Lecture Notes Series, 6, Seoul National University, Research Institute of Mathematics, Global Analysis Research Center, Seoul, 1993, MR 96m:58269, Zbl 0822.58001. 
[9] M.V. Safonov, Harnack's inequality for elliptic equations and Hölder property of their solutions (Russian), Zap. Nauchn. Sem. Leningrad. Otdel. Mat. Inst. Steklov. (LOMI), 96 (1980), 272-287; J. Soviet Math., 21 (1983) 851-863 (English), MR 82b:35045, Zbl 0511.35029.

[10] L. Saloff-Coste, Uniformly elliptic operators on Riemannian manifolds, J. Differential Geom., 36(2) (1992), 417-450, MR 93m:58122, Zbl 0735.58032.

[11] A note on Poincaré, Sobolev, and Harnack inequalities, Internat. Math. Res. Notices, 2 (1992), 27-38, MR 93d:58158, Zbl 0769.58054.

[12] R. Schoen, The effect of curvature on the behavior of harmonic functions and mappings, Nonlinear Partial Differential Equations in Differential Geometry (Park City, UT, 1992), IAS/Park City Math. Ser., 2, Amer. Math. Soc., Providence, RI, 1996, 127-184, MR 96m:53043, Zbl 0852.58023.

[13] R. Schoen and S.-T. Yau, Lectures on Differential Geometry, International Press, Cambridge, MA, 1994, MR 97d:53001, Zbl 0830.53001.

[14] D.W. Stroock, Non-divergence form operators and variations on Yau's explosion criterion, J. Fourier Anal. Appl., 4(4-5) (1998), 565-574, MR 2000b:58066.

[15] S.-T. Yau, Harmonic functions on complete Riemannian manifolds, Comm. Pure Appl. Math., 28 (1975), 201-228, MR 55 \#4042, Zbl 0291.31002.

Received August 2, 2002 and revised January 23, 2003. The author was supported in part by NSF Grant No. DMS-9971052.

SCHOOL OF MATHEMATiCs

UNIVERSITY OF MiNNESOTA

Minneapolis, MN 55455

E-mail address: skim@math.umn.edu 